\title{
On the communication of information by displays of standard errors and confidence intervals
}

\author{
W. K. ESTES \\ Harvard University, Cambridge, Massachusetts
}

\begin{abstract}
A survey of practices regarding the presentation of information about reliability of means in psychological research publications over the last century reveals some advance in quality of communication, greater for tabular presentations than for graphic presentations, but also substantial room for improvement. In this article, problems of interpretation and communication associated with presentations of standard errors and confidence intervals in research reports are examined from both statistical and psychological perspectives. Four general principles of effective communication are proposed and illustrated in application to presentations of data from common psychological research designs, with special attention to problems arising in connection with repeated measures.
\end{abstract}

Contributors to psychological journals are continually admonished that mean values presented in figures or tables should be accompanied by measures of variabilityfor example, standard errors of means (henceforth $S E_{M} \mathrm{~s}$ ) or confidence intervals (CIs). ${ }^{1}$ The advice is unexceptionable, but one may question how well it is followed.

\section{TRENDS IN REPORTING OF VARIABILITY}

Granted that reporting measures of variability and doing it effectively are high-priority objectives for psychological scientists, what do we know about the level and quality of this reporting? I have run across no systematic reviews in the literature, and, to alleviate my curiosity, I engaged in some informal sampling during rest stops in the preparation of this article. Starting with early history, I examined a favorite resource, Ebbinghaus (1885/1913), and found that reporting variability had gotten off to a surprisingly good start, probable errors of means (essentially the same as standard errors) being conscientiously included in nearly all of the large number of tables. A follow-up of that observation showed, however, that it was a flash in the pan. The first 10 volumes of American Journal of Psychology (published over the period 1887-1899) yielded not a single instance of a mean being accompanied by a standard error or equivalent, and the same was true of the first volume (1916) of Journal of Experimental Psychology $(J E P)$. Proceeding more systematically, I scanned each 10 th year of $J E P$ from 1930 through $1990 .^{2}$ Standard errors appeared occasionally in tables in 1930, and the frequency increased steadily until, by 1970 , the inclusion of $S E_{M} \mathrm{~s}$ or standard deviations $(S D \mathrm{~s})$ in tables of means was fairly common. Surprisingly, though, even

Preparation of this article was supported by NSF Grant SBR 9317256 to Harvard University. Correspondence should be addressed to W. K. Estes, Department of Psychology, William James Hall, 33 Kirkland St., Harvard University, Cambridge, MA 02138 (e-mail: wke@) wjh.harvard.edu). in 1990 , a substantial number of tables of means contained no associated measure of variability.

Progress was slower with respect to the display of measures of variability in figures. I found no instances from 1930 through 1960 , and 1970 yielded only one- $-95 \%$ confidence intervals around the best-fitting line through a sequence of plotted means. In $1980, S E_{M}$ bars appeared in a few articles, and, in 1990 (and 1995, which I added for good measure), $S E_{M}$ bars or $C I \mathrm{~s}$ (in both cases, always attached to sample means) appeared in about one article per issue.

It is apparent that the enormous improvement in visual quality of figures and tables published in psychological research journals over the past century has been accompanied by some, though only slowly accelerating, improvement in efficiency of communicating information. The situation is somewhat better for tables than for figures. Tables of means in JEP articles of recent years include measures of variability in a majority of cases. However, the measures are often $S D$ s rather than $S E_{M} \mathrm{~s}$, even when the latter would be much more pertinent to the conclusions under consideration; when data come from complex designs, information as to how the measures were computed is typically scanty or absent, as is any discussion of problems of interpretation of the measures. ${ }^{3}$ With respect to figures, the communication of information about variability associated with means displayed in line or bar graphs has scarcely emerged from the dark ages. The inclusion of $S E_{M}$ s or CIs is still rare, and, when they do appear, the situation is even worse than for tables with respect to the provision of relevant background information.

This brief survey gives rise to two questions: (1) What accounts for the glacial slowness in progress toward uniform and effective communication of information about variability? (2) When measures of variability of means are presented, do they serve their purposes well?

Regarding the first question, several decades of editing journals has led me to think that a great many researchers in psychology do not understand the theoretical and com- 
putational bases of common measures of variability of means well enough to calculate them from the data of commonly used experimental designs, to display them effectively in figures, or to integrate them meaningfully into presentations of results. One reason for this deficiency is that treatments of such common measures as $S E_{M}$ s and $C I s$ in the literature on statistics in psychological research have not kept pace with innovations in research design. For the simplest designs, involving only comparisons of performance by independent groups of subjects, the definition and computation of $S E_{M}$ s usually are clearly described; however, for the very common designs in which repeated measures are obtained on the same subjects, the problem of computing correct estimates of $S E_{M} \mathrm{~s}$, and therefore also $C I \mathrm{~s}$, is rarely mentioned (one of the few exceptions being G. R. Loftus \& E. F. Loftus, 1988).

Regarding the second question, my experience suggests that the incidence of misleading presentations of measures of variability is far from trivial and that it is not notably lower in psychological journals than in others, even though psychologists might be expected to use psychological principles advantageously to guide practice. Textbooks and guidelines for journal contributors indicate that measures of variability should be presented in particular ways - for example, as bars attached to points representing mean scores in figures. But does insertion of a bar in a figure or a number in a table necessarily communicate the desired information to readers? There are several reasons why the answer may be in doubt. (1) Although $S E_{M}$ s and $C I s$ are generated by popular statistical software at the click of a mouse, they convey information only to the degree that both author and reader understand the basis for their computation. Often, a statistic with a given label can be computed from the data of an experiment in several different ways, and the mode used in a particular instance may not be apparent to a reader. (2) For psychological reasons, some forms of presentation may systematically mislead all but the most sophisticated readers. (3) Often, a device is used on different occasions with the same visual appearance but different intended meanings and consequent ambiguity.

In this article, I review some basic characteristics of measures of variability of means (specifically, $S E_{M} \mathrm{~s}$ and $C I$ ), assemble a set of principles that could guide their application in various contexts, and arrive at some recommendations regarding presentation in research reports.

\section{MEASURES OF VARIABILITY OF MEANS: BASIC CONCEPTS}

When they are included in figures, standard error $(S E)$ bands and $C I s$ typically appear indistinguishable to the eye. However, the common visual appearance conceals important differences in the underlying concepts - their histories, their definitions, and their appropriate interpretations. Some of the differences are summarized in Table 1. First, the origins of these concepts are very disparate. The conception of an $S E_{M}$ had been long familiar in physical sciences and engineering (under the label probable error) when it began to infiltrate experimental psychology late in the last century. The reason why $S E_{M}$ has earned relatively widespread usage in psychological research is undoubtedly its relevance to the everyday task of assessing reliability, or replicability, of results. The overweening importance of replicability is perennially highlighted for psychologists. For example, Arthur W. Melton, reflecting on problems and practices of the JEP during his 12 year term as editor, remarked that a principal step in the evaluation of an article always involved "a judgment with respect to the confidence to be placed in the findings - confidence that the results of the experiment would be repeatable under the conditions described" (Melton, 1962, p. 553). A frequent contributor to discussions of methodology, David Lykken, resonated to this theme, "demonstrating an empirical fact must involve a claim of confidence in the replicability of the findings" (Lykken, 1968 , p. 155), then went on to identify a frustrating problem, "Ideally, of course, all experiments would be replicated before publication but this goal is impractical" (Lykken, 1968, p. 159).

The principal difficulty with widespread dependence on replication of experiments is not that conducting replications is tedious ${ }^{4}$ but that judging the success of replications poses an almost intractable problem (Rosenthal, 1993). Happily, although replications are often desirable, sole reliance on them for evidence about replicability is unnecessary. Owing to a remarkable fact of statistical theory, 5 it is possible to compute from the data giving rise to any sample mean a quantity, $S E_{M}$, that provides an estimate of the dispersion of the sample means that would be obtained over a series of replications under the same conditions. The fact that $S E_{M}$ measures dispersion around the true mean, which is nearly always unknown, does not prevent the measure from being extremely useful, but it does set the stage for common misinterpretations, as will be shown in the next section.

The related concept of $\mathrm{Cl}$, a product of relatively modern statistical theory, is less familiar to psychologists. The concept is a derivative of that of $S E$ in that its computation in any instance requires the computation of an $S E_{M}$ as a preliminary step, but it embodies also a statis-

Table 1

Comparison of Standard Error of Mean $\left(S E_{M}\right)$ and Confidence Interval $(\mathrm{CI})$

\begin{tabular}{|c|c|c|}
\hline \multirow[b]{2}{*}{ Property } & \multicolumn{2}{|c|}{ Measure of Variability } \\
\hline & $S E_{M}$ & $\mathrm{Cl}$ \\
\hline Origin & Engineering practice & Statistical theory \\
\hline Purpose & $\begin{array}{c}\text { Judge replicability } \\
\text { of sample mean }\end{array}$ & $\begin{array}{l}\text { Judge goodness of } \\
\text { estimate of } \\
\text { population mean }\end{array}$ \\
\hline $\begin{array}{l}\text { Attribute } \\
\text { measured }\end{array}$ & $\begin{array}{l}\text { Variability of sample } \\
\text { means }\end{array}$ & $\begin{array}{l}\text { Interval including } \\
\text { population mean } \\
\text { with specified } \\
\text { confidence }\end{array}$ \\
\hline $\begin{array}{l}\text { Location } \\
\text { in figure }\end{array}$ & $\begin{array}{l}\text { Centered on population } \\
\text { mean }\end{array}$ & $\begin{array}{l}\text { Centered on sample } \\
\text { mean }\end{array}$ \\
\hline
\end{tabular}


tical inference about the expected frequency with which a given $C I$, if attached to each sample mean over a series of replications, would be found to include the true mean. Problems involved in deriving $S E_{M} \mathrm{~s}$ and $C I$ s for common research designs and in communicating them without hazard of misinterpretation will be a focus of the remaining sections of this article.

Recently, G. R. Loftus and Masson (1994) have made a useful start on this task by reviewing the basic concepts of SEs and CIs and treating some of the problems of computing $C I$ s for repeated measures designs. Here, I go further by discussing some general points of interpretation not covered by G. R. Loftus and Masson, with a focus on the relation between the mode of presentation of information about variability and the hazards of incorrect interpretations.

Considering the task of communicating information about variability from both statistical and psychological perspectives and drawing on several major treatments of graphic display (Kosslyn, 1994; Tufte, 1983; Wainer \& Thissen, 1993), as well as my own experience, I have found that the following general principles provide useful guidelines for treatments of specific problems.

Background: Presentations of measures of variability should include information as to exactly what data the measures are based on, how their computation was accomplished, and what question or issue the measures pertain to.

Consistency: A label or symbol representing a statistic should have the same meaning wherever it occurs.

Compatibility: Symbols and other devices presented in figures should be chosen so that the intended meanings are compatible with the usual habits and expectations of readers. ${ }^{6}$

Cognitive economy: Symbols and other devices (as $S E_{M}$ bars around sample means) should be presented in figures only when they convey information without complicating the interpretive task of the reader.

In the following sections, I illustrate the application of these guidelines to problems that arise, first, in independent-groups designs and, then, in repeated measures designs.

\section{STANDARD ERRORS IN INDEPENDENT-GROUPS DESIGNS}

\section{Modes of Computation}

It will be convenient to point out some issues concerning the interpretation and presentation of $S E S$ by considering samples of data for which population values of means and measures of variability are known. The data curves in the three panels of Figure 1 represent results of a hypothetical experiment in terms of means for five independent groups of 25 subjects who were tested for performance after $1,2,3,4$, or 5 practice trials on a task. The data were produced by a computer program that sampled values from a normal distribution with an $S D$ $(\sigma)$ of .25 and the population trial means shown in the middle panel of Figure 1.
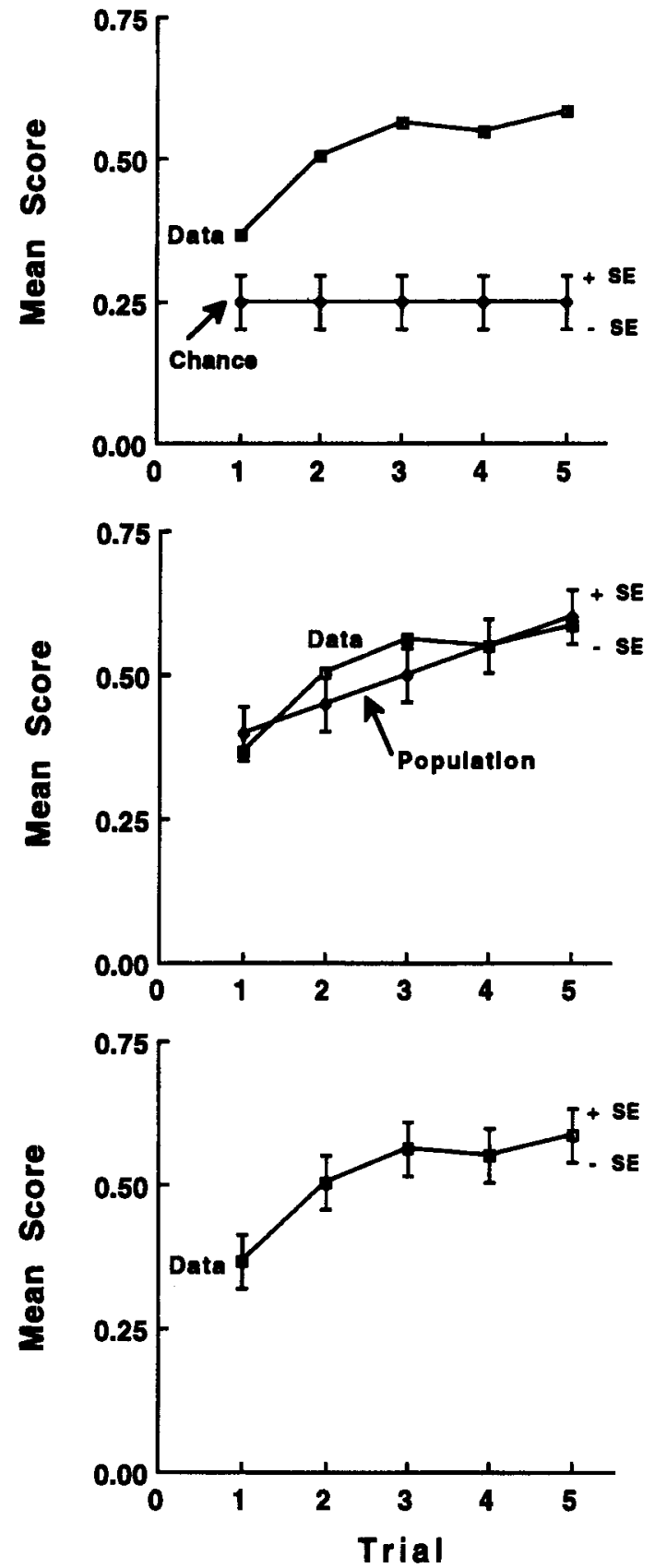

Figure 1. Mean scores for simulated data of five independent groups tested on different trials. Standard error $(S E)$ bars are attached to chance values in the top panel, to population means in the middle panel, and to sample means in the bottom panel.

The top panel of Figure 1 represents a case in which the investigator is interested in how performance following various amounts of practice deviates from a hypothesized baseline (chance) level of .25. The observed trend in the sample means looks orderly, but would it be likely to hold up over replications of the experiment? To approach that question, the now standard procedure is to compute a measure of the variability of the sample means 
for each trial that would be anticipated if the experiment were replicated many times.

From elementary statistics (or see the review by G. R. Loftus \& Masson, 1994), we know that the desired measure depends only on sample size and the way in which the scores sampled vary around their true mean, and this measure can be computed from the simple formula $\sigma_{M}=$ $\sigma / \sqrt{n}$, where $M$ denotes a sample mean, $n$ denotes the sample size, $\sigma$ denotes the $S D$ of scores around the true (population) mean, and $\sigma_{M}$ denotes the $S D$ of sample means around the true mean. In this hypothetical experiment, $n=25$, and $\sigma=.25$, so $\sigma_{M}=.05$. An aspect of the formula for $\sigma_{M}$ that will be critical for later discussions is that it does not depend on the value of the population mean.

When analyzing an experiment in any actual research situation, one does not know the true value of $\sigma$; therefore, one must use an estimate of it based on the observed variability of scores within a sample. The standard measure of this variability is mean square error $\left(M S_{\mathrm{e}}\right)$, computed from the sum of squared deviations of scores in the sample from their mean, and its use for estimation of $\sigma$ derives from the fact that the average value of $M S_{\mathrm{e}}$ is equal to $\sigma^{2}$. In a particular experiment, $M S_{\mathrm{e}}$ may be computed for individual conditions (trials in the example of Figure 1) separately, or a single value may be obtained by pooling error data over conditions, conveniently accomplished in an analysis of variance (ANOVA). Issues relating to the choice of procedures will be discussed later in connection with more complex designs. For purposes of this example, a single estimate of $\sigma$ based on the combined data from all of the observed samples was obtained from an ANOVA on the data for all five trials, which yielded an $M S_{\mathrm{e}}$ of .054 . The square root of this value, .2324 , provides the desired estimate of $\sigma$, and division by the square root of sample size yields $.2324 / 5=.046$ as the estimate of $\sigma_{M}$, a fairly close approximation of the true value. Henceforth, the denotation $S E_{M}$ is reserved for the estimate of $\sigma_{M}$ obtained from data.

\section{Problems of Communication}

The vertical bars of length .046 around the chance value on each trial in the top panel of Figure 1 mark the range within which about two thirds of sample means should be expected to fall over replications of the experiment if the true mean of the population sampled were .25. Observing that all of the sample means fall well above this range, and progressively so over trials, one would conclude (correctly) that a value of .25, constant over trials, for the true means is wildly implausible given the data.

Because we know the values of the population trial means for this hypothetical experiment, it is possible to accompany a plot of the sample means with one of the true means, as done in the middle panel of Figure 1. The $S E$ bars have the same interpretation as before, and it is apparent that the obtained sample means fall almost entirely within the band delineating $\pm 1 S E$ from the true means. An investigator would have been correct in inferring from the data analysis described that the trend of sample means is at least a fair representation of the trend of true means.

In empirical research, investigators do not know the values of true means, and they often have no interest in hypothesized values of true means, so the most common practice is to draw $S E$ bars around observed means, as illustrated in the bottom panel of Figure 1. Because $\sigma_{M}$ and its estimator, $S E_{M}$, refer to variability of sample means around true means, the interpretation that the $S E$ bars show the range within which about two thirds of the sample means should be expected to fall in replications of the experiment is incorrect. ${ }^{7}$ To point up this fact, $\mathrm{I}$ have plotted in Figure 2 the sample mean from Trial 3 of Figure 1 together with the frequency distribution of sample means generated in 100 replications of the hypothetical experiment. It is apparent that the replication means cluster symmetrically around the true mean with a majority falling within $\pm 1 S E_{M}$, however, a much smaller percentage of replication means falls within $\pm 1 S E_{M}$ of the sample mean. The distribution of replication means around the sample mean is highly asymmetric, and its $S D$ clearly is larger than the $S E_{M}$.

It is easy to generalize from this example and show that in general the variability of replication means around a sample mean is larger than their variability around the true (population) mean (Estes, 1991). Whereas the $S D$ of replication means around the population mean, $\mu$, is given by the square root of $\sigma^{2} / \sqrt{n}$, the $S D$ around a sample mean, $M_{0}$, is given by the square root of the quantity $\left(\sigma^{2} / n\right)+\left(\mu-M_{0}\right)^{2}$, which is larger, unless $M_{0}=\mu$, and may be much larger. Consequently, $S E_{M}$, the standard estimator of $\sigma_{M}$, underestimates the $S D$ of replication means around the sample mean.

One basis for the persisting practice of drawing $S E$ bars around sample means may be the thought that, because a sample mean is the best estimator of the population mean, the quantity $\mu-M_{0}$ will usually be small and the

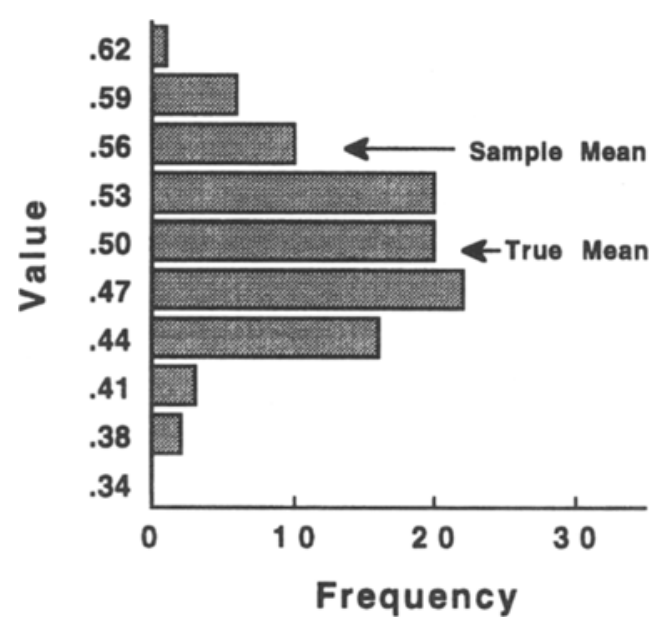

Figure 2. Frequency distribution of sample means in 100 replications of Group 3 of the simulated experiment represented in Figure 1. The arrows indicate the sample mean (.56) for Trial 3 of Figure 1 and the true mean of the population sampled (.50). 
$S D$ of replication means around $M_{0}$ will usually be close to the $S D$ around $\mu$. However, the thought is misguided. To illustrate what may actually be expected, Figure 3 shows how the difference between the $S D$ s of replication means around the sample mean and around the population mean varies as a function of the disparity between $\mu$ and $M_{0}$. For each of two sample sizes, 10 and 25, the horizontal line represents the theoretical $S D$ around the population mean, $\sigma_{M}$, and the rising curve represents the $S D$ of replication means around the sample mean at each value of $\mu-M_{0}$; the vertical difference between corresponding points on the rising curve and the $\sigma_{M}$ line for the given condition signifies the error in taking $S E$ bars around the sample mean as a measure of variability of replication means around the sample mean. The points are plotted at steps of $\sigma / 4$ along the abscissa, so it is apparent that disparities of $\sigma / 2$ or more between $\mu$ and $M_{0}$, which occur with appreciable frequency, will produce substantial errors.

It is straightforward to go beyond examples and derive an explicit expression for the $S D$ of the distribution of replication means around a sample mean. We note that, if the distribution of scores is normal, the deviation of a replication mean, $M_{\mathrm{r}}$ from the sample mean, $M_{0}$, is the difference between two variables, each of which is normally distributed with mean $\mu$ and variance $\sigma^{2} / n$. From a general theorem concerning linear combinations of normally distributed variables (Mood, 1950), it follows that the variance of $M_{\mathrm{r}}-M_{0}$ is the sum of the variances of $M_{\mathrm{r}}$ and $M_{0}$. Thus, the variance of replication means around the sample mean is simply $\sigma^{2} / n+\sigma^{2} / n=2 \sigma^{2} / n$, and the $S D$ is approximately $1.4 \sigma / \sqrt{n}$. Furthermore, so long as samples are drawn independently, this result holds gen-

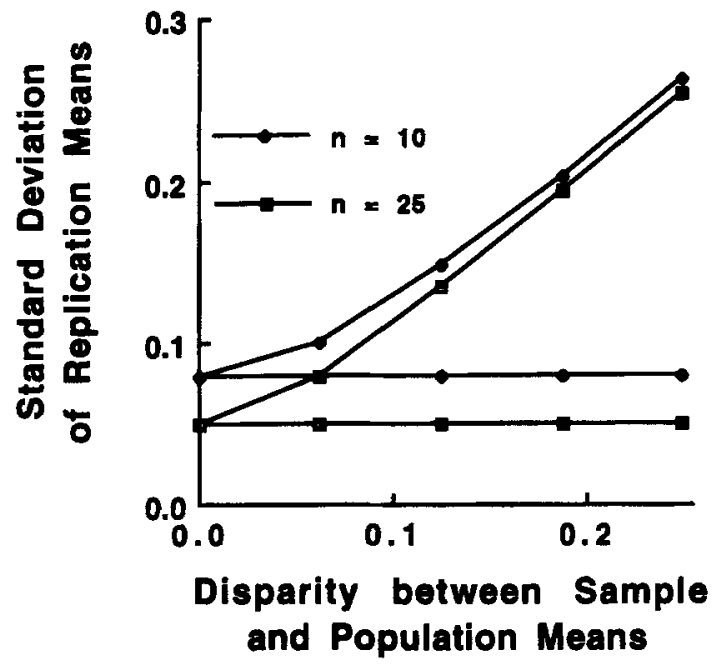

Figure 3. Standard deviation of replication means around the current sample mean (rising curves) and around the population mean (horizontal lines) as a function of the disparity between the current sample mean and the population mean. The population standard deviation, $\sigma$, for the distribution of scores giving rise to the functions is equal to .25 ; the parameter $\boldsymbol{n}$ denotes sample size. erally, even if the distribution of scores is not normal (Appendix A).

One of the main purposes of plotting sample means together with $S E$ bars is to give a visual impression of the trend to be expected in replications of an experiment. However, the fact that the impression is often inaccurate and sometimes grossly misleading seems not to be well known. Consequently, there is reason for reservations about the common practice of printing these plots as they come from statistical programs without justification of their use and their intended interpretation in individual cases. The practice of plotting confidence intervals around sample means involves some additional considerations and will be discussed in a later section.

\section{STANDARD ERRORS IN REPEATED MEASURES DESIGNS}

\section{Modes of Computation}

In this section, I take a step beyond the treatment of repeated measures in G. R. Loftus and Masson (1994) by examining in greater depth a groups $\times$ trials design that is very commonly employed in research on learning, retention, generalization, and transfer and that brings up some additional considerations concerning presentation of standard errors. As an example for detailed analysis, I generated a data set for a hypothetical experiment in which two groups of 25 subjects are tested on five successive trials. Mean performance curves for the two groups are shown in Figure 4, and, just as in the case of independent samples, questions arise as to what measures of variability might appropriately be displayed along with the means.

An approach sometimes seen in published studies is simply to use any standard statistical program to compute $S E_{M}$ estimates for each group on each trial from the formula $S E_{M_{\mathrm{ij}}}=S D_{\mathrm{ij}} / \sqrt{n}$, where $S D_{\mathrm{ij}}$ denotes the sample standard deviation for Group $i$ on Trial $j$. The results for this example are shown in Table 2. However, unless there is reason to believe that population $S D$ s are very heterogeneous across groups or trials, it is generally considered preferable to obtain a single estimate of $S E_{M_{\mathrm{ij}}}$, denoted $S E_{M_{\mathrm{GT}}}$, based on the data from all cells of the design - that is, all group $\times$ trial (GT) combinations. ${ }^{8}$ To enable computation of global estimates of this and other statistics for this design, it is necessary to conduct a repeated measures ANOVA on the simulated data.

The model for the design of the hypothetical experiment, basic to both the program that generated the simulated data and to interpretation of the ANOVA, takes the form

$$
Y_{\mathrm{ijk}}=\mu+\alpha_{\mathrm{i}}+\beta_{\mathrm{j}}+\alpha \beta_{\mathrm{ij}}+P_{\mathrm{k}: \mathrm{i}}+\beta P_{\mathrm{jk}: \mathrm{i}}+e_{\mathrm{ijk}},
$$

where $Y_{\mathrm{ijk}}$ represents the score for Subject $\mathrm{k}$ in Group $\mathrm{i}$ on Trial j; $\mu$ represents the mean score for the population sampled; $\alpha_{i}$ and $\beta_{\mathrm{j}}$ represent the effects associated with membership in Group $\mathrm{i}$ and testing on Trial $\mathrm{j} ; \alpha \beta_{\mathrm{ij}}$ represents the interaction effect for Group $\mathrm{i}$ on Trial $\mathrm{j} ; P_{\mathrm{k}: \mathrm{i}}$ 

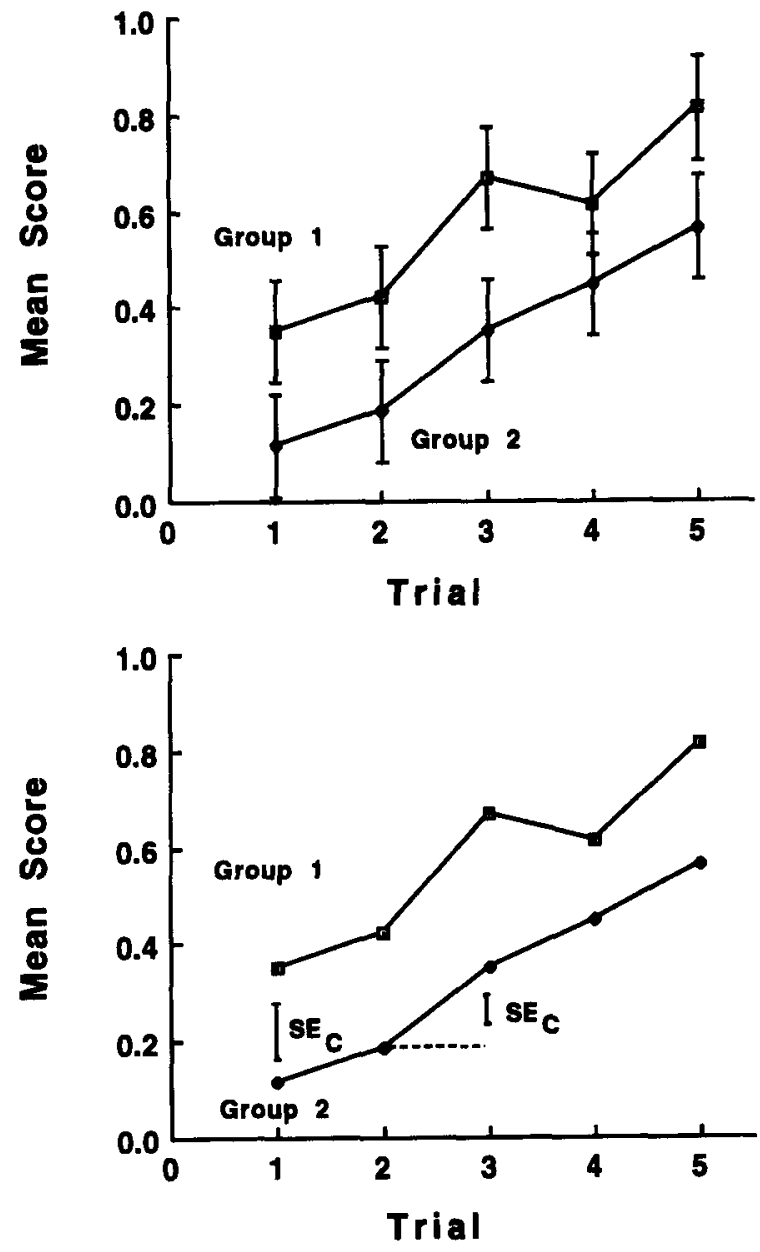

Figure 4. Mean score by trial for two groups in a hypothetical experiment with a repeated measures design. In the upper panel, $68 \%$ confidence intervals have been centered on the points representing trial means for each group. Standard errors for group means on any trial, for contrasts comparing group means on any trial, and for contrasts comparing any group mean with the mean for that group on another trial are $.106, .150$, and .076 , respectively (the latter two being shown graphically by the bars labeled $S E_{C}$ in the lower panel).

represents the mean score (over all trials) for Subject $\mathrm{k}$ in Group i; $\beta P_{\mathrm{jk}: \mathrm{i}}$ represents the interaction effect associated with testing of Subject $k$ of Group $i$ on Trial $j$; and $e_{i j k}$ represents the random error associated with testing of Subject $k$ of Group $i$ on Trial $j$.

Table 3 summarizes the expected mean squares that are derived from Equation 1 and that are required to guide the computation of significance tests and expressions for $S E$ s.

The terms $\sigma_{P}^{2}, \sigma_{T(P: G)}^{2}$, and $\sigma^{2}$ in Table 3 are the variances of the random effects in the design (i.e., the last three terms on the right side of Equation 1). The terms involving $\theta$ are measures of the fixed effects of the design, defined as

$$
\theta_{\alpha}^{2}=\left(\Sigma \alpha_{\mathrm{i}}^{2}\right) /(s-1)
$$

$$
\theta_{\beta}^{2}=\left(\Sigma \beta_{\mathrm{j}}^{2}\right) /(t-1)
$$

and

$$
\theta_{\alpha \beta}^{2}=\left(\Sigma \alpha \beta_{\mathrm{ij}}^{2}\right) /(s-1)(t-1),
$$

where $s$ and $t$ denote the numbers of groups and trials, respectively. All of these terms are discussed, and derivations of the expected mean squares (EMSs) in Table 3 are given in Estes (1991).

A quantity that is important for computation of $S E s$ the pooled mean square within cells of the design, $M S_{\mathrm{W}}-$ does not appear in the table of mean squares (nor in the ANOVA output listing) but can be computed from the relation

$$
M S_{\mathrm{W}}=\left[M S_{P: G}+(t-1) M S_{\mathrm{T}(P: \mathrm{G})}\right] / t
$$

(Estes, 1991; Winer, 1971).

To generate the simulated data, convenient values were chosen for the fixed quantities in Equation 1, and values for the variable quantities were produced by a random number generator. The table of scores so obtained ${ }^{9}$ was entered in a program for an ANOVA assuming fixed effects of groups and trials and repeated measures over trials, the output being summarized in Table 4 .

With the results of the ANOVA in hand, we are ready to compute a single value of the $S E$ of a sample mean for

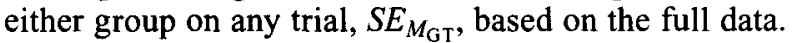
This computation and related ones can be understood in terms of the expressions for the relevant population and sample statistics summarized in Table 5. The center column presents population variances of means and contrasts, and the rightmost column presents corresponding estimators of them in the form of squared $S E$ s that are computable from the indicated data mean squares. As seen in the top segment of Table 5, the estimate of $S E_{M_{\mathrm{GT}}}$ is based on the pooled mean square within cells, $M S_{\mathrm{W}}$, defined in Equation 2 and obtainable from the mean squares in the lowermost two rows of Table 4 . The corresponding population value is computed similarly from the expected mean squares in Table 3 with the result shown in the top segment of Table 5; as should be expected, the estimate from the data proves to be quite close to the population value. ${ }^{10}$

In general, a useful rule of thumb is that one will nearly always be on sound ground if one uses the mean square

Table 2

Standard Errors for Repeated Measures Example Based on Data of Each Group on Individual Trials

\begin{tabular}{llllll}
\hline & \multicolumn{5}{c}{ Trial } \\
\cline { 2 - 6 } Group & 1 & 2 & 3 & 4 & 5 \\
\hline 1 & & & & & \\
$M$ & .35 & .42 & .67 & .62 & .81 \\
$S E_{M}$ & .10 & .10 & .09 & .11 & .10 \\
2 & & & & & \\
$M$ & .12 & .19 & .35 & .45 & .56 \\
$S E_{M}$ & .11 & .11 & .11 & .12 & .10 \\
\hline Note-M $-M=$ mean; $S E_{M}=$ standard error of mean.
\end{tabular}


Table 3

Expected Mean Squares for Groups $\times$ Trials Design

\begin{tabular}{|c|c|c|c|}
\hline Source & $d f$ & Mean Square & Expected Mean Square \\
\hline $\mathrm{G}$ & $s-1$ & $M S_{\mathrm{G}}$ & $n t\left(\theta_{\alpha}\right)^{2}+t \sigma_{P}^{2}+\sigma^{2}$ \\
\hline $\mathrm{T}$ & $t-1$ & $M S_{\mathrm{T}}$ & $n s\left(\theta_{\beta}\right)^{2}+\sigma_{\mathrm{TP}: \mathrm{G}}^{2}+\sigma^{2}$ \\
\hline GT & $(s-1)(t-1)$ & $M S_{\mathrm{GT}}$ & $n\left(\theta_{\alpha \beta}\right)^{2}+\sigma_{T P: G}^{2}+\sigma^{2}$ \\
\hline$P: \mathrm{G}$ & $s(n-1)$ & $M S_{P: \mathrm{G}}$ & $t \sigma_{P}^{2}+\sigma^{2}$ \\
\hline $\mathrm{T}(P: \mathrm{G})$ & $s(t-1)(n-1)$ & $M S_{\mathrm{T}(P: \mathrm{G})}$ & $\sigma_{\mathrm{T}_{(P: \mathbf{G})}^{2}}^{2}+\sigma^{2}$ \\
\hline
\end{tabular}

Table 4

Analysis of Variance on Artificial Repeated Measures Data

\begin{tabular}{lrc}
\hline Source & $d f$ & Mean Square \\
\hline $\mathrm{G}$ & 1 & 3.63 \\
$\mathrm{~T}$ & 4 & 1.69 \\
$\mathrm{GT}$ & 4 & 0.04 \\
$P: \mathrm{G}$ & 48 & 1.12 \\
$\mathrm{~T}(P: \mathrm{G})$ & 192 & 0.07 \\
\hline
\end{tabular}

Note- $\mathrm{G}=$ groups; $\mathrm{T}=$ trials; $P: \mathrm{G}=$ subjects in groups; $\mathrm{T}(P: \mathrm{G})=$ trials $\times$ subjects in groups.

within cells, $M S_{\mathrm{W}}$, pooled over all cells of the design, as the basis for estimating the $S E$ of a cell mean, $S E_{M_{\mathrm{GT}}}$, for both independent-groups designs and repeated measures designs. With respect to $S E$ s of contrasts (comparisons) between means, when the means of different groups on any one trial are being compared, the basis should be the pooled $M S_{\mathrm{W}}$, but when the comparison involves means for any one group on different trials, the basis should be the appropriate mean square for interaction of the relevant experimental factor with subjects-within-groups, as shown in the middle and bottom sections of Table 5.

\section{Problems of Communication}

How the $S E_{M_{\mathrm{GT}}}$ value obtained from a repeated measures ANOVA can best be communicated depends on one's purposes. When the intent is only to convey information about the probable replicability of observed sample means, it is customary to include the $S E_{M_{\mathrm{GT}}}$ value in a footnote to a table of means or to attach corresponding $S E$ bars ( \pm .106 in the repeated measures example) to the points in a figure representing sample means - for example, the points plotted for both groups in Figure 4. This tactic is subject to the same reservations discussed for the independent-groups example in relation to Figure 1, and, at the least, the $S E_{M_{\mathrm{GT}}}$ value should be multiplied by a factor of 1.4 to correct for the systematic underestimation of the true variability of replication means demonstrated above.

When one's interest is in how well population means have been estimated by sample values, $S E$ bars based on the obtained $S E_{M_{\mathrm{GT}}}$ can be appended to theoretical values, as was done for the chance values in the top panel of Figure 1 and might be done for the repeated measures case in Figure 4. However, there is a problem of interpretation. When the sample means for successive values of the independent variable, trials in Figures 1 and 4, come from repeated measures on the same subjects, then, according to the model for the analysis, deviations of the trial means from corresponding population values arise not only from random error (the term $e_{\mathrm{ijk}}$ in Equation 1) but also from subject effects $\left(P_{\mathrm{k}: \mathrm{i}}\right.$ and $\beta P_{\mathrm{jk:i}}$ in Equation 1) that are correlated over trials. This correlation needs to be taken into account when one draws any conclusion from the pattern of sample means over trials because the means will deviate consistently in the same direction from the population means with higher probability than would be the case for independent groups that yielded the same $S E_{M}$ bars.

When comparisons of the means of different groups on any one trial are at issue, the $S E$ of a comparison (contrast), $S E_{C}$, is more informative with respect to the reliability of a group difference than the $S E$ of a sample mean. Thus, the likelihood of appropriate interpretation will be enhanced if the presentation of the $S E_{M_{\mathrm{GT}}}$ from the ANOVA in a table of trial means or inclusion of $S E$ bars based on this value in a figure is accompanied by mention of the value of the corresponding $S E_{C}$ for such comparisons (defined in the middle panel of Figure 5) in a footnote to the table or the caption of the figure.

When one's interest is in the patterns of group means over trials, then neither of the $S E_{M}$ values given in the upper and middle panels of Table 5 is informative because neither takes account of the trial-to-trial correlation that must be expected in a repeated measures design. The value of $S E_{C}$ for a comparison between sample means on different trials is directly relevant, and its value proves to be much smaller than the value for comparisons of different groups on individual trials (as may be seen in the rightmost column of Table 5). It is certainly appropriate to report $S E_{C}$, but a display in the form of $S E$ bars around sample means has no straightforward interpretation. A tactic that might be considered is to display an $S E$ bar of length equal to the $S E_{C}$ estimate but dissociated from the sample mean curves, as illustrated in the lower panel of Figure 4. Better, perhaps, would be to cite the values of $S E_{M_{\mathrm{GT}}}$ and the $S E_{C}$ s defined in the middle and bottom sections of Table 5 in the figure caption, as done in the caption for Figure 4.

Table 5

Standard Errors for Cell Means in Repeated Measures Example

\begin{tabular}{|c|c|c|}
\hline Statistic & Population Value & Estimate From Data \\
\hline $\begin{array}{l}\text { Group mean } \\
\text { on a trial }\end{array}$ & $\begin{aligned} \sigma_{M_{\mathrm{GT}}}^{2} & =E M S_{\mathrm{W}} / n \\
& =.298 / 25 \\
& =.0119 \\
\sigma_{M_{\mathrm{GT}}} & =.109\end{aligned}$ & $\begin{aligned} S E_{M_{\mathrm{GT}}}^{2} & =M S_{\mathrm{W}} / n \\
& =.282 / 25 \\
& =.0113 \\
S E_{M_{\mathrm{GT}}} & =.106\end{aligned}$ \\
\hline $\begin{array}{l}\text { Comparison of } \\
\text { group means } \\
\text { on a trial }\end{array}$ & $\begin{aligned} \sigma_{C}^{2} & =2 E M S_{\mathrm{W}} / n \\
& =.0238 \\
\sigma_{C} & =.154\end{aligned}$ & $\begin{aligned} S E_{C}^{2} & =2 M S_{\mathrm{W}} / n \\
& =.0226 \\
S E_{C} & =.150\end{aligned}$ \\
\hline $\begin{array}{l}\text { Comparison of } \\
\text { means of group } \\
\text { on different trials }\end{array}$ & $\begin{aligned} \sigma_{C}^{2} & =2 E M S_{\mathrm{T}(P ; \mathrm{G})} / n \\
& =2(.050) / 25 \\
& =.004 \\
\sigma_{C} & =.063\end{aligned}$ & $\begin{aligned} S E_{C}^{2} & =2 M S_{\mathrm{T}(P \cdot \mathrm{G})} / n \\
& =2(.072) / 25 \\
& =.006 \\
S E_{C} & =.076\end{aligned}$ \\
\hline
\end{tabular}




\section{CONFIDENCE INTERVALS}

Especially in theoretically oriented research, sample means are of interest mainly for the information they yield about the values of population means. It is well known that a sample mean is the best estimator of a population mean, but it is desirable to accompany this estimate with a measure of its precision. The most commonly used measure is the $C I$, or confidence band, the basis for which has been presented in the discussion of the concept of $S E_{M}$ at the beginning of this article. If, in any experiment, samples of size $n$ are drawn from a population of scores with mean $\mu$ and standard deviation $\sigma$, the sample means will be distributed at least approximately normally around $\mu$ with $\sigma_{M}$ equal to $\sigma / \sqrt{n}$. The distribution shown in Figure 2, for example, comes from drawing samples of size 25 from a population with a mean of .50, $\sigma$ of .25., and, therefore, $\sigma_{M}$ of .05. Referring to a normal curve table, we can determine that a sample mean will fall within, say, one $S E$ of the true mean - that is, within a band of length $2 \times .05=.10$ centered on .50 , with probability .68 . It follows that, with the same probability, a band of length .10 centered on any sample mean includes the true mean, .50 . Consequently, given a particular sample mean, for example the mean of .56 marked in Figure 2, one denotes the band of length . 10 around .56, as a $68 \%$ confidence band, or $C I$, and the endpoints of the interval as confidence limits.

In actual research, one does not know the value of $\sigma_{M}$, but the explication of the concept of $C I$ just given is unchanged if one replaces $\sigma_{M}$ with its estimate, $S E_{M}$, computed from the data. Thus, in the example of Figure 2, the sample yielding the observed mean of .56 has a sample $S D$ of .177 and, therefore, an $S E_{M}$ equal to .055 , so a $68 \% C I$ would be of length .11 .

Conventionally, $\mathrm{CIs}$ are computed for probabilities ( $C$ values) associated with commonly used significance levels and expressed as percentages (e.g., $C$ equal to $95 \%$ or $99 \%$, for the .05 and .01 significance levels) rather than for probabilities corresponding to $\sigma_{M}$ or $S E_{M}$ units. When the sample size is small, probabilities are obtained from the $t$ rather than the normal distribution. ${ }^{11}$ These technicalities are discussed in many elementary texts and are thoroughly reviewed by G. R. Loftus and Masson (1994). Here, I wish only to emphasize the basic point that the concept of $C I$ is based on the distribution of sample means around the population mean, and a $C I$ around a sample mean conveys information not about the likelihood that replication means will fall within the interval but only about the likelihood that, over a series of similar occasions, the population mean will be found to fall within the interval.

When sample means come from a repeated measures design, as the example in Figure 4, $S E_{M}$, computed in the manner described in the previous section, can be used to construct a $C I$ for any GT mean just as in the case of independent groups. In Figure 4, $68 \% \mathrm{CIs}$ (i.e., intervals of length $2 \times S E_{M}$ ) have been centered on each of the GT means. Considering any one GT mean by itself, the interpretation is the same as in the case of independent groups: Over a series of replications of the experiment, the $C$-level $C I$ around the mean of a group on any one trial will contain the corresponding population mean on a percentage $C$ of occasions. Thus, just as in the case of independent groups, the $C I$ provides an indicator of the precision with which the sample mean, $M_{\mathrm{GT}}$, estimates the population mean, $\mu_{\mathrm{GT}}$.

This $C I$ is useful without qualifications mainly to facilitate comparisons between experiments. For example, if plots of the form of those in Figure 4 were obtained from several experiments of the same design conducted with different experimental parameters, the CIs would provide valid indicators of the relative precision of the $\mu_{\mathrm{GT}}$ estimates obtained from different experiments. However, when one's interest is in the pattern of GT means for a group over trials in any one experiment, an interpretation of the CIs in terms of probabilities must take account of the nonindependence of scores for a group over trials inherent in the repeated measures design.

When interest is specifically in comparing the means for a particular group on two or more trials, the appropriate error estimate (the square root of $M S_{\mathrm{T}(P: \mathrm{G})} / n$ ) can be entered in the formula for the $S E$ of a contrast, as shown in the bottom section of Table 5 and used to construct CIs. G. R. Loftus and Masson (1994) describe the procedure in detail and suggest that these intervals, which, of course, will be different from the CIs computed for individual GT means, be attached to the GT means in a groups $\times$ trials graph. Some reservations about that practice will be discussed below.

\section{RECOMMENDATIONS}

In this article, the problem of presenting information about variability effectively has been addressed from two perspectives: statistical and psychological. G. R. Loftus and Masson (1994) give useful discussions of statistical problems having to do with the measures that are available for various purposes and how the measures can be computed for research designs more complex than factorial combinations of independent groups. I have supplemented their treatment at a few points, but my central focus has been on the psychological question of how best to serve the user.

\section{Background Information}

Even in as routine a situation as including $S E$ s in a table of means, the issue of presenting adequate background information is not trivial. Consider, for example, Table 2. As might often be done, the $S E_{M}$ s were computed by a simple descriptive statistics program that used the within-cell mean square $\left(M S_{\mathrm{W}}\right)$ for each group on each trial as the basis for the $S E_{M}$ accompanying the cell mean; not unexpectedly, the patterns of $S E_{M}$ values over trials differ slightly for the two groups. If, however, $S E_{M} \mathrm{~s}$ had been requested from the program that computed the 
ANOVA for the experiment, the appearance of the table would have been different: On each trial, the $S E_{M}$ s for both groups would have been identical $(.10, .09, .10, .11$, and .10 for each group on Trials $1-5$, respectively). The reason for the different result in the second computation is that the ANOVA program bases the $S E_{M}$ s on the pooled $M S_{\mathrm{W}}$ for each column obtained from subsidiary ANOVAs for the simple effect of groups on individual trials. If I had chosen to depart from the program and compute a single $S E_{M}$, applicable to all of the cells in the table, I would have based the computation on a pooled $M S_{\mathrm{W}}$ obtained from the full data of the experiment in the manner discussed in an earlier section.

Often, in actual research, differences among the estimates obtained in these different ways are much larger than in my hypothetical example; for readers concerned with comparisons of experiments or with aggregating results of experiments, as in meta-analyses, it is important to know the mode of computation used in each case. The thought that the need for full disclosure could be eliminated by imposing uniformity of the mode of computation for all research published in a given journal must be dismissed out of hand, because, as well brought out by G. R. Loftus and Masson (1994), the advantages and disadvantages of the different modes differ importantly with circumstances. Choosing the mode of computation requires informed judgment in each individual case. When the choice has been made, information about the mode chosen and any special considerations entering into the decision should accompany the presentation of results, whether the measures of variability are given in the text of an article or displayed in tables or figures.

\section{Displaying Standard Errors}

Centering $S E_{M}$ bars or $C I$ s on points representing sample means in figures can be an effective way of communicating information about variability, but special attention to the principles of information display is needed in order to minimize hazards of misinterpretation. These two types of display involve some different issues and need separate consideration.

As brought out in the earlier discussion, the display of $S E_{M}$ bars around population means (e.g., chance levels of performance or means predicted by theoretical models ${ }^{12}$ ) is often a useful practice. Attachment of $S E_{M}$ bars to plotted sample means, however, invites misinterpretation. A natural interpretation, and perhaps the one assumed implicitly by all but the most statistically sophisticated journal readers, is that an $S E_{M}$ bar gives information about the reliability of a sample mean by indicating the range within which about $68 \%$ of sample means should be expected to fall over replications of the experiment. The pernicious feature of this interpretation is that it is in part well founded but in part unfounded. The phrasing of this interpretation commonly seen in textbooks-for example, "the interval defined by the sample mean $\pm 2 S E$ generally contains about $95 \%$ of the means (not the individuals) that would be observed if samples of the same size were repeatedly selected" (Dawson-Saunders \& Trapp, 1994, p. 86), or "Error bars indicate the range over which measurements are likely to vary with repeated sampling" (Kosslyn, 1994, p. 59) - is ambiguous in a major respect: Error bars indicate the length, but not the location, of the interval on the vertical dimension of a figure within which $68 \%$ of replication means will fall. Referring again to the hypothetical experiment represented in Figure 1, the $S E_{M}$ bars of length .046 attached to the displayed mean for Trial 3 suggest that about $68 \%$ of replication means should fall between .514 and .606 ; however, because the value .046 estimates the $S D$ of replication means around the population mean (in this case, .50), the correct expectation is that about $68 \%$ of replication means should fall between .454 and .546 . It is the latter expectation that fits the actual distribution of replication means shown in Figure 2. Furthermore, as brought out in the first section of this article, the length of the interval around the sample mean that should contain about $68 \%$ of replication means is not .092 (twice the $S E_{M}$ value of .046) but that value corrected by a factor of 1.4 . Thus, if $S E_{M}$ bars are displayed with the purpose of conveying information about replicability of sample means, the purpose should be stated explicitly, and the values displayed should be corrected for the underestimation.

Though the choice of procedure for displaying measures of variability must allow for individual differences in style among investigators, I personally recommend that $S E_{M}$ bars not be attached to plotted sample means. The reason, referring back to the consistency principle, is that $S E_{M}$ bars and $C I s$ around sample means look alike but have distinctly different interpretations. The same problem is not acute when $S E_{M} \mathrm{~s}$ are included in tables of means, because $C I$ s rarely appear in such tables, and, in any event, labels for the measures included appear in row or column headings. Even when $S E_{M}$ s are presented in tables, however, if they are used to support conclusions about the probable replicability of the sample means, it must be appropriate to take some account of the systematic bias inherent in the use of $S E_{M}$ s for that purpose.

\section{Displaying Confidence Intervals}

When $\mathrm{Cl}$ s are presented in figures, they are necessarily centered on plotted sample means. If the means are for independent groups, the interpretation is straightforward. However, when there is any possible ambiguity, the presentation should be accompanied by an explicit specification of the population means whose probable locations are indicated by the $C I s$. If the means come from a repeated measures design - for example, the one giving rise to Figure 4-the same comments apply, but it is even more important to give full information about how the $C I$ s are computed and what inferences they pertain to, because there is no standardization in either respect.

For example, G. R. Loftus and Masson (1994) emphasize that, in the case of groups $\times$ trials curves coming from repeated measures designs, the investigator may be interested in the pattern of a group's means over trials rather than 
in the location of particular population means, and that $C I s$ based on within-cell mean squares $\left(M S_{\mathrm{W}}\right.$ in Table 4$)$ are not relevant to this purpose. G. R. Loftus and Masson give a clear exposition of how one can derive quasi-CIs, based on interaction rather than within-cell mean squares, that are relevant to such comparisons and suggest that these be presented in figures to aid judgments about the patterning of trial-to-trial variation. ${ }^{13}$ Unfortunately, this tactic runs afoul of several principles of effective communication. Quasi-CIs centered on sample means in a figure look exactly like actual $C I$ s but they do not have the same interpretation: the latter give information about the range within which the population mean corresponding to the sample mean is likely to fall, whereas the former bear on comparisons between a particular sample mean and means for the same group on other trials.

In the interest of consistency and unambiguity of communication, it seems preferable to exhibit only actual $C l s$ in figures and to do so only in connection with the issue of how closely population means are estimated by obtained sample means. Questions about patterning can be addressed in other ways. For example, if the derivations supplied by G. R. Loftus and Masson (1994) are used to construct actual $\mathrm{Cls}$ around contrasts among means for a group on different trials, these CIs can be interpreted in the standard manner to convey information about the range within which population values of the contrasts are likely to fall. For an example, consider the pattern of means for one of the groups - say, Group 2 -represented in Figure 4. A contrast that might be of interest is the slope of a linear regression line fitted to the means. The estimated slope proves to be .116 and its standard error .013 (details of the computation being given in Appendix $B$ ), so expressing the result in the conventional form $.116 \pm .013$ would communicate $68 \%$ confidence that the true slope falls between .103 and .129 .

A property of $C I s$ that may interfere with felicitous communication of information is the inherent association of a $C I$ with a particular choice of significance level. As discussed above (see note 11), the length of a $C I$ is obtained by multiplying the appropriate error estimate by two times the criterion value of $t$ for the significance level corresponding to the chosen confidence level (e.g., the criterion value of $t$ for .05 significance when the confidence level is to be $95 \%$ ). The principle of consistency implies that, within any one research report, the Cls exhibited in all figures should have the same interpretation, a requirement that can be met only if a single significance level is adopted for all tests performed. That practice is widely recommended, but the issue is debatable, and investigators often deviate from the recommended practice. (Note the frequency of "* $=.05$ level; $* *=.01$ level" and the like in published ANOVA tables).

An additional problem with displaying $C I$ s representing different confidence levels in the same article, or even in related articles, has to do with compatibility. A $99 \% C l$, of course, implies higher likelihood than a $95 \%$
CI that a population mean falls within a given range; however, attached to a sample mean in a figure, a $99 \% \mathrm{Cl}$ is larger than a $95 \% C I$ and tends to give the impression that the location of the population mean is less sharply constrained. When different $\mathrm{Cl}$ s appear in different figures that are being compared, conflicting connotations add to the processing demand on the reader. ${ }^{14}$

A way of combining consistency with convenience and, at the same time, eliminating the compatibility problem is to dissociate the use of $C I s$ as surrogates for significance tests from their use as conveyers of visual information about the precision of estimates of parameters. The dissociation could be achieved by following a standard practice of using $C I s$ corresponding to conventional significance levels, such as $95 \%$ or $99 \%$, when the CIs appear in the text of an article in reference to comparisons of means but using $C I$ s corresponding to $S E_{M}$ units for display in figures when the purpose is simply to give a visual impression of the precision with which a population mean has been estimated. For the simulated experiment represented in Figure 4, for example, one might exhibit $68 \% C I$ bars in the figure, as has been done in the upper panel, to serve the latter purpose. ${ }^{15}$ Even though the data come from a repeated measures design, the bars represent genuine CIs, based on the $S E_{M}$ defined in the top panel of Table 5. However, for the purpose of assessing the significance of contrasts between means, either across groups or across trials, $C /$ s for the desired confidence level would be computed from the $S E$ s of contrasts defined in the lower two panels of Table 5 .

\section{CONCLUDING COMMENT}

In a recent article (Estes, 1997), I noted that the majority of articles presenting research results in contemporary psychological journals include tests of statistical significance, usually presented in a stereotyped summary of significance of main effects of experimental factors and their interactions based on ANOVAs or similar analyses. Taking that observation together with the survey reported at the beginning of this article, one might conclude that the analysis of data, or at least the communication of results, too often stops with the display of sample means in tables or figures together with citations of significance levels that provide virtually no help to readers concerned with understanding in detail the story that a set of data has to tell. The response to this unsatisfactory state of affairs suggested by the treatments of problems of communication in this article is not that investigators should be advised to dispense with ANOVAs and the like but, rather, that they should be encouraged to draw on the riches contained in the outputs of those global analyses for the ingredients of measures of variability of means or comparisons among them that are directly informative regarding estimates of replicability of results and inferences from sample statistics to population values or parameters of theoretical models. 


\section{REFERENCES}

Dawson-Sanders, B., \& TraPp, G. (1994). Basic and clinical biostatistics (2nd ed.). Norwalk, CT: Appleton \& Lange.

Ebbinghaus, H. (1913). Memory (H. A. Ruger \& C. E. Bussenius, Trans.). New York: Columbia University, Teachers College Press. (Reprint New York: Dover, 1964; Original work published 1885)

ESTES, W. K. (1991). Statistical models in behavioral research. Hillsdale, NJ: Erlbaum

EsTES, W. K. (1993). Mathematical models in psychology. In G. Keren \& C. Lewis (Eds.), A Handbook for data analysis in the behavioral sciences: Methodological issues (pp. 3-19). Hillsdale, NJ: Erlbaum.

ESTES, W. K. (1997). Significance testing in psychological research: Some persisting issues. Psychological Science, 8, 18-20.

Kosslyn, S. M. (1994). Elements of graph design. New York: Freeman. LofTUs, G. R. (1993). Editorial comment. Memory \& Cognition, 21, 1-3. LoFTUs, G. R., \& LofTUs, E. F. (1988). Essence of statistics (2nd ed.). New York: Knopf.

LoFTUS, G. R., \& MASson, M. E. J. (1994). Using confidence intervals in within-subject designs. Psychonomic Bulletin \& Review, 1, 476-490

LYKKEN, D. T. (1968). Statistical significance in psychological research. Psychological Bulletin, 70, 151-159.

MELTON, A. W. (1962). Editorial. Journal of Experimental Psychology, 64, 553-557.

Mood, A. M. (1950). Introduction to the theory of statistics. New York: McGraw-Hill.

NEELY, J. H. (1995). Editorial. Journal of Experimental Psychology: Learning, Memory, \& Cognition, 21, 261.

RosenthaL, R. (1993). Cumulating evidence. In G. Keren \& C. Lewis (Eds.), A handbook for data analysis in the behavioral sciences. Methodological issues (pp. 519-559). Hillsdale, NJ: Erlbaum.

SNedecor, G. W., \& Cochran, W. G. (1980). Statistical methods (7th ed.). Ames, IA: Iowa State University Press.

TAYLOR, J. R. (1982). An introduction to error analysis. Mill Valley, CA: University Science Books.

TUFTE, E. R. (1983). The visual display of quantitative information. Cheshire, CT: Graphics Press.

WAINER, H. (1996). Visual revelations: Scaling the heights (and widths) Chance, 9, 43-49.

WAINER, H., \& THISSEN, D. (1993). Graphical data analysis. In G. Keren \& C. Lewis (Eds.), $A$ handbook for data analysis in the behavioral sciences: Statistical issues (pp. 391-457). Hillsdale, NJ: Erlbaum.

WINER, B. J. (1971). Statistical principles in experimental design. New York: McGraw-Hill.

\section{NOTES}

1. See, for example, Publication Manual of the American Psychological Association (4th ed.), "where means are reported, always include an associated measure of variability" (p. 15); G. R. Loftus (1993), "By default, data should be conveyed as a figure depicting sample means with associated standard errors" (p. 3); Neely (1995), "In reporting results, authors should still provide measures of variability. ... There are a number of acceptable ways to do this, including reporting $M S E$ s or confidence intervals" (p. 261).

2. The 1980 and 1990 volumes were Journal of Experimental Psychology: Learning, Memory, \& Cognition.

3. Be it understood that I am not aiming criticism at the $J E P$, which, I am sure, would rate among the best of research journals in any broader survey; it was chosen for illustrative purposes only because I have most of the volumes used conveniently at hand on my office shelves.

4. Replicating experiments is unpopular as well as tedious. During his $J E P$ editorship, Melton established a section of the journal for brief Replication Reports, which yielded few published reports and ultimately faded away.

5. Clearly, well known to statisticians by the middle of the 19th century, though none of the histories I have been able to consult identifies the originator

6. This principle has been expressed cogently by Wainer (1996):
A graph is nothing but a visual metaphor. To be truly evocative, it must correspond closely to the phenomenon it depicts; longer bars or bigger pie slices must correspond to more; a rising line must correspond to an increasing amount. If a graphic depiction of data does not faithfully follow this notion, it is almost sure to be misleading. (p. 43)

7. This common but unsound interpretation apparently derives in part from seemingly authoritative statements that are to be found in the physics and engineering literature-for example, "measured value of time $=2.4 \pm 0.1 \mathrm{sec}$. $[0.1 \mathrm{sec}$. being one $S E]$ is exactly equivalent to the two statements 'best estimate of time $=2.4 \mathrm{sec}$.' and 'probable range, 2.3 to 2.5 sec.' " (Taylor, 1982, p. 15).

8. The former procedure has the advantage of not depending on assumptions concerning homogeneity of variances and correlations of errors, the latter being the advantage of the greater stability attained by having a single estimate based on many more observations than any of the single-trial estimates. This tradeoff is discussed by G. R. Loftus and Masson (1994)

9. Details of the simulation can be obtained from the author on request (preferably via e-mail).

10. The estimate based on $M S_{\mathrm{W}}$ is the most convenient one available, though not quite optimal. The variance of scores within cells, $\sigma_{\mathrm{GT}}^{2}$, is the sum of the variances of the random effects that contribute to the variability of the constituent scores and can be computed from $\sigma_{\mathrm{GT}}^{2}=\sigma_{P}^{2}+$ $\sigma_{\mathrm{T}(P: \mathrm{G})}^{2}+\sigma^{2}$. Substitution of the known values of these variances yields $\sigma_{\mathrm{GT}}^{2}=.30$, and therefore, $\sigma_{M_{\mathrm{CT}}}^{2}=\left(\sigma_{\mathrm{GT}}^{2}\right) / 25=.012$ and $\sigma_{M_{\mathrm{GT}}}=.1095$, very close to the value based on $M S_{\mathrm{W}}$. Estimates of the three population variances are not obtainable from the ANOVA output, so, in practice, one uses the estimate of $\sigma_{M G T}^{2}$ based on $M S_{\mathrm{W}}$.

11. A level $C$ confidence interval is computed from $C I=2 t^{*} / M S_{\mathrm{e}} / n$, where $t^{*}$ is the criterion value of $t$ for significance level $1-C$ with the appropriate degrees of freedom for the given design, $n$ is the number of scores in each sample, and $M S_{\mathrm{e}}$ is the appropriate error estimate (the mean square within cells, $M S_{\mathrm{W}}$, for the case of independent groups).

12. Strictly, the predictions should be parameter-free--that is, derived without use of any information from the data-as is true, for example, of predictions about probability matching (Estes, 1993).

13. G. R. Loftus and Masson (1994) also consider the problem of compatibility between the conclusions suggested by a figure and by an ANOVA on the data represented in the figure. They show how this compatibility can be improved for a repeated measures design by plotting means for scores transformed by an adjustment that eliminates intersubject variance.

14. Tufte (1983, p. 153) points up the principle involved: "The complexity of multifunctioning elements can sometimes turn data graphics into visual puzzles, crypto-graphical mysteries for the viewer to decode. ... By contrast, in a non-puzzle graphic, the translation of visual to verbal is quickly learned, automatic, and implicit."

15. This tactic can be conveniently implemented because many commercially available statistics/graphing programs now allow the user to choose from a menu the length of $C I$ bars to be plotted, and at least one (Statview II, Abacus Concepts, Inc., 1987) instructs the user how to select $C I$ bars corresponding to $S E_{M}$ units.

\section{APPENDIX A \\ Derivation of Variance of Replication Means Around a Sample Mean}

In an experiment, a sample of $n$ scores is drawn from a population of scores having mean $\mu$ and standard deviation $\sigma$, then, in replications of the experiment, a series of additional samples of size $n$ is drawn from the same population. Denoting the mean of the first sample by $M_{0}$ and any replication mean by $M_{\mathrm{r}}$, we wish to obtain the variance of $M_{\mathrm{r}}$ around $M_{0}$.

By definition, the desired variance is equal to the population mean of $\left(M_{\mathrm{r}}-M_{0}\right)^{2}$, that is, $\mathrm{E}\left[\left(M_{\mathrm{r}}-M_{0}\right)^{2}\right]$, where $\mathrm{E}$ denotes mathematical expectation. The quantity $M_{\mathrm{r}}-M_{0}$ is obviously 
equal to $\left(M_{\mathrm{r}}-\mu\right)-\left(M_{0}-\mu\right)$, so the variance expression can be expanded in the identity

$$
\begin{aligned}
\mathrm{E}\left[\left(M_{\mathrm{r}}-M_{0}\right)^{2}\right]= & \mathrm{E}\left[\left(\left(M_{\mathrm{r}}-\mu\right)-\left(M_{0}-\mu\right)\right)^{2}\right] \\
= & \mathrm{E}\left[\left(M_{\mathrm{r}}-\mu\right)^{2}+\left(M_{0}-\mu\right)^{2}\right] \\
& -2 \mathrm{E}\left[\left(M_{\mathrm{r}}-\mu\right)\left(M_{0}-\mu\right)\right] .
\end{aligned}
$$

The quantities $\mathrm{E}\left[\left(M_{\mathrm{r}}-\mu\right)^{2}\right]$ and $\mathrm{E}\left[\left(M_{0}-\mu\right)^{2}\right]$ are the variances of $M_{\mathrm{r}}$ and $M_{0}$, each equal to $\sigma^{2} / n$, and the expectation of the cross product $\mathrm{E}\left[\left(M_{\mathrm{r}}-\mu\right)\left(M_{0}-\mu\right)\right]$ can easily be shown to be zero, so we arrive at the simple result

$$
\mathrm{E}\left[\left(M_{\mathrm{r}}-M_{0}\right)^{2}\right]=\sigma^{2} / n+\sigma^{2} / n=2 \sigma^{2 / n},
$$

and the population standard deviation of replication means around the sample mean is $\sigma / \sqrt{n}$, approximately equal to $1.4 \sigma / \sqrt{n}$.

\section{APPENDIX B \\ Computation of Confidence Intervals for a Contrast}

Starting with a brief review of relevant basics, a contrast among a set of means, $M_{\mathrm{j}}$, is given by

$$
C=\sum \lambda_{\mathrm{j}} M_{\mathrm{j}},
$$

where the $\lambda_{\mathrm{j}}$, the contrast weights, are numerical coefficients that sum to 0 , and the squared standard error of a contrast is given by

$$
S E_{C}^{2}=M S_{\mathrm{e}} \sum \lambda_{\mathrm{j}}^{2} / n,
$$

where $n$ is the number of scores entering into any mean, and $M S_{\mathrm{e}}$ is the appropriate mean square error (Snedecor \& Cochran, 1980). For the particular case of a linear regression function for a set of means, the slope of the line is a particular case of a contrast, defined by $\beta=\sum_{\mathrm{j}} M_{\mathrm{j}} x_{\mathrm{j}} / \sum x_{\mathrm{j}}^{2}$, and $x_{\mathrm{j}}=X_{\mathrm{j}}-M_{x}$, where $X_{\mathrm{j}}$ is the value of the independent variable associated with sample $\mathrm{j}$, and $M_{x}$ is the mean of the $X_{\mathrm{j}}$. This expression is obtained from Equation $\mathrm{A} 1$ by letting $\beta=C$ and $\lambda_{\mathrm{j}}=x_{\mathrm{j}} / \sum x_{\mathrm{j}}^{2}$.

For the example discussed in the text concerning the curve of means for Group 2 in Figure 4, the values of $X_{\mathrm{j}}$ (trials) are 1, 2, 3,4 , and 5 , yielding $x_{j}$ equal to $-2,-1,0,1$, and 2 , respectively, $\sum x_{j}^{2}$ equal to 10 , and $\lambda_{j}$ equal to $-.2,-.1,0, .1$, and .2 . Inserting the $\lambda_{\mathrm{j}}$ values together with the observed sample means in Equation $A 1$ yields .116 as our estimate of $\beta$. The same result could be obtained by entering the data into any statistical program for a simple regression analysis. The $M S_{\mathrm{e}}$ needed to compute the standard error of $\beta$ is not generated by any commercial statistical program that I am familiar with, but it can easily be obtained by running a repeated measures ANOVA on the same data, which, in this instance, yields an $M S_{\mathrm{e}}$ equal to .045 . (Note that the $M S_{\mathrm{e}}$ produced by a regression program is a residual term that includes a mixture of error, as defined in the model for the experiment, and disparities between scores predicted by the regression function and the observed scores. The procedure used in this example, and generalizable to other designs, amounts to modifying the standard ANOVA by partitioning the sum of squares for the relevant experimental factor [trials in the example] into a component associated with the linear contrast-that is, the regression function--and a remainder [Estes, 1991]). Entering this value, together with $n$ equal to 25 and the $x_{\mathrm{j}}$ calculated above into Equation 2 yields $S E_{\beta}^{2}=.00018$ and, therefore, $S E_{\beta}=.013$.

(Manuscript received December 20, 1996; revision accepted for publication April 28, 1997.) 